\title{
CHARACTERIZATION OF SPARC PHYSIOLOGICAL FUNCTION BY IMMUNE-INHIBITED GROUPER
}

\author{
Yi-Tien Chen ${ }^{1,2}$, Chao-Fen Lin ${ }^{1,2}$, Tzong-Yueh Chen ${ }^{1,2,3,4} \S$ \\ ${ }^{1}$ Institute of Biotechnology, National Cheng Kung University, Tainan 70101, Taiwan \\ ${ }^{2}$ Department of Biotechnology and Bioindustry Sciences, National Cheng Kung University, \\ Tainan 70101, Taiwan \\ ${ }^{3}$ Translational Center for Marine Biotechnology, National Cheng Kung University, Tainan \\ 70101, Taiwan \\ ${ }^{4}$ Agriculture Biotechnology Research Center, National Cheng Kung University, Tainan 70101, \\ Taiwan
}

\begin{abstract}
Secreted protein acidic and rich in cysteine (SPARC) involves antigen-specific immune responses of dendritic cell migration. It is also a matricellular protein that correlates with adipocyte hyperplasia and hypertrophy and regulates adipogenesis and lipid accumulation in muscle. However, the physiological function of fish SPARC remains unclear. Therefore, the objective of this study is to characterize SPARC physiological function by immune-inhibited groupers. The immune-inhibition was performed by triggering the adaptive immune response against SPARC in orange-spotted grouper. After the treatment, the adaptive immune response was represented by the value of anti-SPARC titer. The SPARC-inhibited grouper showed higher value than control in various parameters including body weight, body weight without viscera and fillet weight. In addition, the fatty acid composition of muscle SPARC-inhibited grouper is similar to control groupers. Although none of effect shown on total serum cholesterol, the serum triglyceride significant increased. The adipocyte density and lipid accumulation increased in muscle tissue. Furthermore, it is interesting that SPARC mRNA expression in muscle tissue was increased while the protein expression of SPARC decreased. In conclusion, the grouper SPARC regulates the adipogenesis and lipid accumulation without modification of fatty acid composition and then further affects the growth of the grouper. These results provide a new model system by immuneinhibition technology for physiological function analysis in fish.
\end{abstract}

\section{KEYWORDS}

grouper, SPARC, immune-inhibition, adaptive immune, growth performance

${ }^{\S}$ Corresponding author. Tel.: +886-6-2757575\#65622-610; Fax: +886-6-2766505

E-mail address: ibcty@mail.ncku.edu.tw 